\title{
THE SEPARATION OF OXIDASE REACTIONS FROM THE CATALASE REACTION
}

G. B. $R E E D$

\section{(WITH THREE FIGURES)}

It is an interesting fact that, with one or two exceptions, the oxidases of plant and animal tissue, even when purified by the best existing methods, still give the catalase reaction, that is, decompose hydrogen peroxide with the evolution of oxygen in its molecular or relatively inactive form. The relation of this catalase action to the physiological activity of the organism is not understood, although several explanations have been advanced.

LOEW $^{\mathrm{I}}$ was the first to isolate a body capable of decomposing hydrogen peroxide but possessing no other enzyme properties. This body he called catalase. Many investigations since this time have proved the correctness of LoEw's results. SENTER ${ }^{2}$ prepared from blood a catalase free from haemoglobin or oxidases. LIEBERMANN $^{3}$ has shown that aqueous extracts of the mesenteric fat of hogs and rabbits decompose hydrogen peroxide without showing any oxidase reactions. Catalase in every way similar to that isolated by LOEW has also been prepared by WENDER ${ }^{4}$ from yeast, and by PAzZI-EscoT ${ }^{5}$ from various higher plants.

As a result of his observations, Loew (loc. cit.) reached the conclusion that catalase is universally distributed, occurring not only in every organism but in every living cell. He assumed, therefore, that it must have some definite function. Since hydrogen peroxide results from the oxidation of many readily oxidizable substances, he regarded it as at least conceivable that this compound may be produced in the living cell as a result of respiratory processes. The accumulation of such a substance would doubtless

${ }^{1}$ LoEw, O., Report no. 68. U.S. Dept. Agric. p. 47. rgor.

${ }^{2}$ Senter, G., Zeit. Physikal. Chem. 44:257-263. 1903; 51:673-705. 1905.

${ }^{3}$ Liebermani, L., Pflüger's Archiv. ro4:1 19-233. 1904.

${ }^{4}$ Wender, Chem. Zeit. 28:300-301, 322-323. I904.

${ }_{5}^{5}$ Pazzi-Escot, Bull. Soc. Chem. Par. 27:280-288. I902. 3०3]

[Botanical Gazette, vol. 62 
prove harmful, and its destruction by catalase would not only prevent injury, but the oxygen liberated would be used again in respiration.

Usher and PRIESTLY ${ }^{6}$ believe that hydrogen peroxide is formed in relatively large amounts in photosynthetic processes, and that its decomposition by catalase prevents it from reaching a sufficient concentration to bleach the chlorophyll.

On the other hand, SHAFFER ${ }^{7}$ considers that the decomposition of the peroxide is of advantage principally in checking oxidation processes, basing his theory on the fact that the oxygen liberated from the decomposition is a poorer oxidizing agent than the hydrogen peroxide. In this connection BATTELLI and STERN ${ }^{8}$ state that catalase retards the oxidation of ferrous sulphate in the presence of animal tissue.

There is very little experimental evidence, however, to indicate that hydrogen peroxide is actually produced in living tissue, except under specialized conditions. Moreover, it seems to be not particularly toxic, since the writer has found that wheat, rice, and radish seedlings develop without ill effects in as high concentrations as $0.5^{-\mathrm{I}}$ per cent. The hypothesis that catalase is of advantage in checking oxidations is also largely invalidated by the observation of $\mathrm{BACH}$ and $\mathrm{CHODAT}^{9}$ that catalase acts only on hydrogen peroxide, and not on organic peroxides or on the oxygenases; for it is these oxygen compounds rather than hydrogen peroxide that are concerned in respiratory oxidations. The writer has found also that while colloidal platinum decomposes hydrogen peroxide, it has no action on the oxygenases. An extract of the outer tissue of potatoes, which exhibits an active oxygenase in its direct action on gum guaiac (free from peroxide), was shaken for some hours with colloidal platinum, but after removing the platinum by filtration ${ }^{\text {10 }}$

${ }^{6}$ Usher, F. L., and Priestly, J. H., Proc. Roy. Soc. London 77:369-376, 78: $318-327$.

7 Shaffer, P. A., Amer. Jour. Physiol. 14:299-312. I905.

${ }^{8}$ Battelli, F., and Stern, L., Compt. Rend. Acad. Sci. I4I:9i6-9I8. I904.

9 Bach, A., and Chodat, R., Ber. Deutsch. Chem. Gesells. 37: 1342. I904.

ro This sample of colloidal metal could be removed from its solution by passing through a hard filter paper; this is shown by the fact that the filtrate from a watery suspension had no action on hydrogen peroxide. 
it was found that the oxygenase was quite as active as before the treatment.

From these observations it appears that the function of catalase in protecting the organism against hydrogen peroxide is extremely limited, if indeed it may be said to exist at all.

Finally, it has been suggested many times, and supported by a considerable body of experimental data, that the action of catalase is associated with the reactions of the oxidases. Although the isolation of catalase has shown that the oxidases are not of necessity concerned in the decomposition of hydrogen peroxide, as was originally supposed, the idea is still held generally that the action of the oxidases is in some manner connected with or dependent upon the catalase reaction.

This association of oxidase action with the decomposition of hydrogen peroxide was suggested at an early date by ScHoenbein. He found that plant and animal tissues were usually capable of effecting the decomposition of hydrogen peroxide and activating many oxidation processes. In studying the relative power of various organs of the animal body to break down hydrogen peroxide, SPITZER $^{\text {II }}$ found them to stand in about the same order in which ABELOUS and BIARNÈs ${ }^{\mathrm{I} 2}$ had arranged them in respect to their ability to oxidize salicylic aldehyde. From this correlation SpItzer was led to believe that both processes have the same cause, and he, therefore, used the rate of evolution of oxygen from hydrogen peroxide as a measure of the oxidizing ability of tissues and tissue extracts.

These observations have been extended by LoEvenHaRT and KASTLE, ${ }^{13}$ who have also studied the behavior of certain inorganic catalyzers in this connection. They report a striking parallelism between the rate at which a number of substances activate the oxidation (by hydrogen peroxide) of formic acid or of formic aldehyde, and the rate of hydrogen peroxide in the presence of these substances. They accordingly conclude that catalase action

in Spitzer, W., Pflüger's Archiv. 67:615-656. I897.

12 Abelous and Biarnès, Arch. de Physiol. 8:31 I. I896.

${ }^{13}$ Loevenhart, A. S., and Kastle, J. H., Amer. Chem. Jour. 29:397-437, 563588. 1903 . 
and the activation by enzymes of oxidations in the presence of hydrogen peroxide (peroxidase action) are closely correlated and may be referred to a common cause.

A study of the relations existing between the catalase and oxidase actions of colloidal platinum and of certain plant extracts has led the writer to a different conclusion. The present paper contains an account of the experiments with colloidal platinum.

In a previous paper $^{14}$ it was pointed out that the direct oxidase action (that is, in the absence of peroxides) of colloidal platinum, is dependent upon the amount of oxygen combined with it; that the reaction can be completely inhibited by electrolytic reduction, and again renewed by recharging the platinum with oxygen at an anode. It was also pointed out that the peroxidase action is essentially the same process; namely, in the presence of hydrogen peroxide the metal is kept fully charged with oxygen, while it is at the same time actively giving up oxygen to the substance undergoing oxidation.

The amount of oxygen which could be combined with the platinum electrically was only sufficient to bring about a very small amount of oxidation as compared with that effected by a low concentration of hydrogen peroxide. It was expected, therefore, that the amount of oxygen in combination with the platinum, when it was introduced into a solution of hydrogen peroxide and an oxidizable substance, would have very little effect, either on the rate of reaction or on the amount oxidized. Several measurements proved this to be the case.

Two platinum disks of exactly the same size, $2 \mathrm{~cm}$. in diameter, were cut from the same piece of foil, sealed into glass tubes for convenience in handling, and platinized by the usual method. Care was taken to continue the platinizing for the same length of time with the same current, so that equal amounts of colloidal platinum were deposited on each electrode. These were then attached to opposite poles of a I Io-volt circuit delivering one ampere and connected by placing in the opposite arms of a $U$ tube containing dilute salt solution. In this way one electrode was subjected to active oxygen, the other to active hydrogen.

${ }^{14}$ Reed, G. B., Bot. Gaz. 62:53-64. figs. 4. I916. 
The peroxidase activity of the two preparations was then compared by removing them from the electrolytic apparatus, washing them quickly by dipping them several times into a considerable volume of distilled water, and placing them sepa- o rately in flasks, each of which contained Ioo cc. of the following mixture: o. I M formaldehyde, $0.03 \mathrm{M} \mathrm{NaOH}$, and approximately o. $\mathrm{IM} \mathrm{H}_{2} \mathrm{O}_{2}$. The solutions were kept well stirred and at a constant temperature of $18^{\circ}$ C. on a water bath. At frequent intervals portions consisting of $2 \mathrm{cc}$. each were removed and titrated with $0.05 \mathrm{M} \mathrm{HCl}$ for the amount of formic acid produced from the oxidation of the formaldehyde. The results are indicated in fig. I, where the curve representing the action of the oxidized platinum is seen to be identical with that which represents the action of the reduced platinum. The points indicated by an $\odot$ represent the reaction of oxidized platinum, and the points indicated by a $\times$ represent the reac-

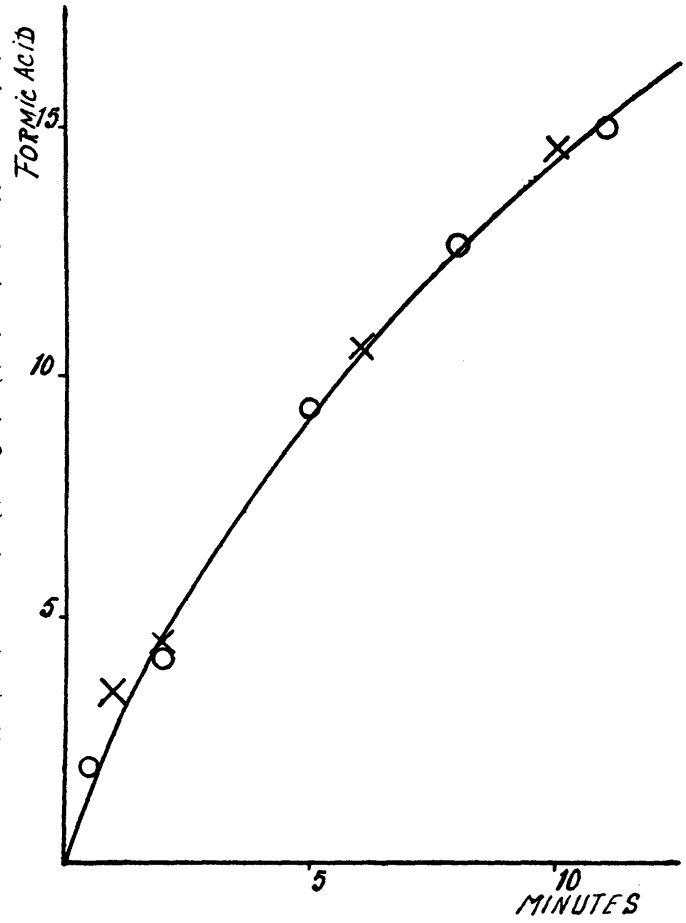

FIG. I.-Curve showing oxidation of formaldehyde by hydrogen peroxide in presence of colloidal platinum: points indicated by $\odot$ are from an experiment in which the platinum had been subjected to active oxygen just previous to placing it in the formaldehyde mixture; points indicated by $\times$ are from a second experiment in which the platinum had been previously subjected to active hydrogen; ordinates represent amounts of formic acid formed (expressed as the difference in number of cc. of $0.05 \mathrm{M} \mathrm{HCl}$ required at the beginning and during the course of the experiment); abscissae represent time in minutes.

tion of reduced platinum. It is thus apparent that the two preparations of platinum are equally effective in this oxidation. Oxidation 
(in the presence of hydrogen peroxide) of potassium iodide, pyrogallol, and SpITzER's reagent was likewise found to be equally catalyzed by platinum, whether it had been treated with active

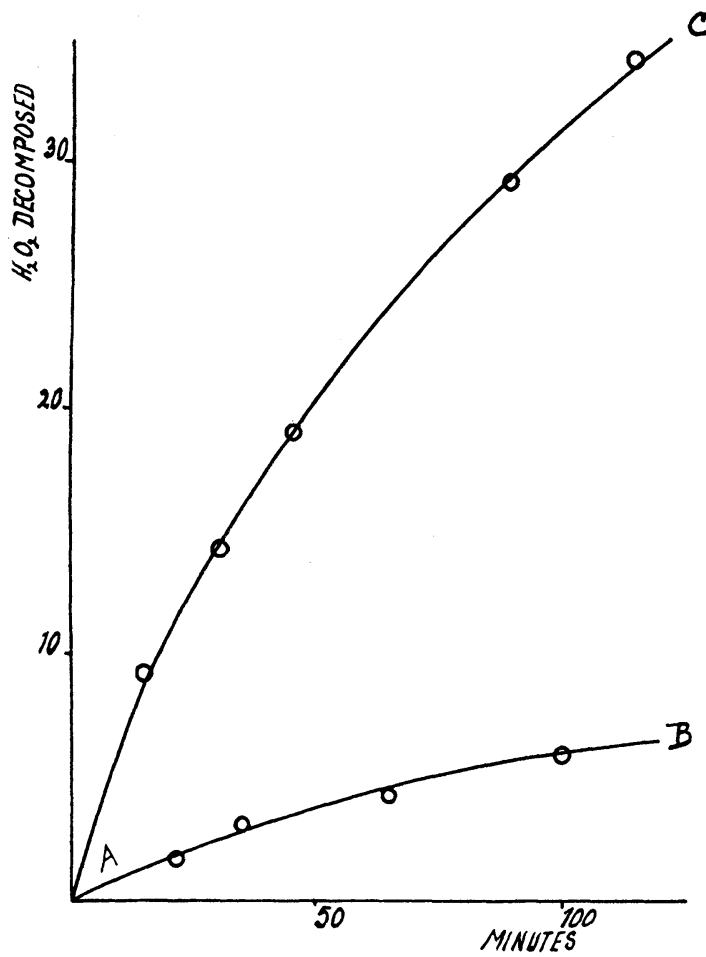

FIG. 2.-Curves showing decomposition of hydrogen peroxide: $A B$ shows activity of platinum black which had previously been subjected to active oxygen by using it as an anode; $A C$ shows action of platinum black which had previously been subjected to active hydrogen by using it as a cathode; ordinates represent percentage of hydrogen peroxide decomposed; abscissae represent time in minutes. c oxygen or with hydrogen.

The action of platinum black in decomposing hydrogen peroxide, however, was greatly modified by its previous treatment. The two electrodes, freshly platinized and subjected to active oxygen and hydrogen as before, were placed separately in flasks, each of which contained roo cc. of o. IM hydrogen peroxide, to which a mixture of phosphates $(5 \mathrm{cc}$. of o. IM $\mathrm{NaH}_{2} \mathrm{PO}_{4}$ and Io cc. o. IM Na $\mathrm{NPO}_{4}$ ) had been added in order to preserve the neutrality of the solution. As the decomposition of the peroxide proceeded, portions consisting of $2 \mathrm{cc}$. each were pipetted out, acidified with $\mathrm{H}_{2} \mathrm{SO}_{4}$ and titrated with potassium permanganate for the amount of hydrogen peroxide left in the solution. ${ }^{\text {is }}$ The results are shown in fig. 2, where $A B$ indicates

is Since hydrogen peroxide reduces acidified potassium permanganate quantitatively, the amount of the latter used in a titration is a measure of the unchanged peroxide present in the solution. EULER (Hofmeister's Beiträge 7:1. 1908) has employed this method in the study of catalase. 
the rate at which hydrogen peroxide is decomposed by platinum black which had previously been subjected to active oxygen (by using it as an anode), and $A C$ indicates the rate obtained with platinum black which had previously been subjected to active hydrogen (by using it as a cathode); it is apparent that the latter treatment greatly accelerates the catalase action. Thus while electrolytic oxidation or reduction of the platinum apparently has no effect on the peroxidase action of the metal, the catalase action is very greatly modified. That this treatment has no effect on the peroxidase action may be explained by following the oxidation potential of the platinum during the reaction. In a previous paper (loc. cit.) it was pointed out that in oxidations by hydrogen peroxide, where colloidal platinum played the part of a peroxidase, the peroxide served to maintain the potential on the

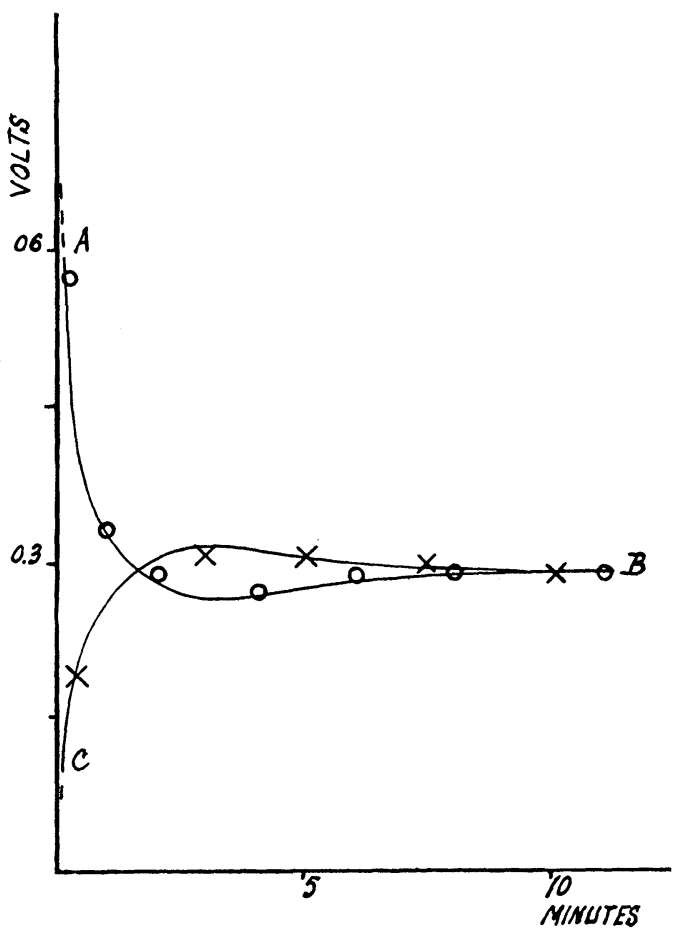

FIG. 3.-Curves of oxidation potential of a platinum cathode in a $0 . \mathrm{rM}$ solution of hydrogen peroxide: upper curve $A B$ represents drop in potential on an electrode which had previously been treated with active oxygen by using it as an anode; lower curve $C B$ represents rise in potential on an electrode which had previously been subjected to active hydrogen by using it as a cathode; ordinates represent volts; abscissae, time in minutes. catalyzer at a maximum, although the substance undergoing oxidation would tend to pull down the potential. If this be true (since previous treatment with either active hydrogen or oxygen has no effect on the peroxidase action) the same potentials should be 
reached in the peroxide solution, whatever the previous condition of the platinum. The following experiment shows this to be the case.

One of the platinized electrodes which had previously been treated with active oxygen, was connected to the system: $\mathrm{Hg}-$ $\mathrm{Hg}_{2} \mathrm{Cl}_{2}-\mathrm{KCl}-\mathrm{H}_{2} \mathrm{O}_{2}-\mathrm{Pt}$, and the oxidation potential determined. ${ }^{16}$ The hydrogen peroxide solution was of the same concentration as used in the previous decomposition experiments (о. IM), and contained a similar solution of phosphates to preserve neutrality. The rate of drop in potential is shown by the curve $A B$, fig. 3. The platinum electrode was then treated with active hydrogen and placed in a similar solution of hydrogen peroxide. In this case the initial potential was low but rapidly increased, as shown by $C B$, fig. 3 , to the same equilibrium as reached in the previous case. By comparing these curves it will be apparent that the previous treatment has no effect on the potential assumed by the platinum after it has been a short time in the peroxide.

Although the potential changes on the platinum indicate why previous oxidation or reduction should not alter the peroxidase activity, it makes more complicated the problem of the changes in catalase activity. It is apparent, however, that factors which have no effect on the peroxidase activity do alter the catalase action. In other words, the peroxidase action is quite independent of the rate of hydrogen peroxide decomposition, and the two reactions may be regarded as quite separate. Further experiments by the writer indicate that a similar separation of the oxidase from the catalase reaction may occur in the living cell. These experiments will be discussed in a subsequent paper.

\section{Laboratory of Plant Physiology}

HARVARD UNIVERSITY

${ }^{16}$ ReED, G. B., Bot. Gaz. 6r:523-527. figs. 2. I9r6. 